\title{
Toward understanding the multiscale spatial spectrum of solar convection
}

\author{
A. V. Getling, ${ }^{1}$ O. S. Mazhorova ${ }^{2}$ and O. V. Shcheritsa ${ }^{2}$ \\ ${ }^{1}$ Institute of Nuclear Physics, Lomonosov Moscow State University, Moscow, Russia, \\ email: A.Getling@mail.ru \\ ${ }^{2}$ Keldysh Institute of Applied Mathematics, Moscow, Russia, \\ email: olgamazhor@mail.ru, shchery@mail.ru
}

\begin{abstract}
Convection is simulated numerically based on two-dimensional Boussinesq equations for a fluid layer with a specially chosen stratification such that the convective instability is much stronger in a thin subsurface sublayer than in the remaining part of the layer. The developing convective flow has a small-scale component superposed onto a basic large-scale roll flow.
\end{abstract}

Keywords. Sun: interior, convection

The multiscale structure of solar convection, i.e., the coexistence of cellular flows sharply differing in their spatial scale, has not yet received a convincing explanation. It is known that the convection cells whose horizontal size is small compared with the layer thickness should also be confined in the vertical direction to a relatively thin sublayer (height interval). This can easily be imagined if a certain sublayer in which convection can occur due to an unstable thermal stratification contacts another sublayer where the stratification is stable and suppresses convective motions (in this case, the flow nevertheless involves the stable region, i.e., penetrative convection occurs). If, however, the layer is convectively unstable throughout its whole thickness, the confinement of flows to a relatively thin sublayer is a much less trivial effect, expecially if large cells filling the whole layer thickness are also present.

Out aim is to comprehend how the peculiarities of the thermal stratification can give rise to a multiscale spectrum of convection provided this stratification is convectively unstable at all heights. It can naturally be assumed that the splitting of scales could result from sharp changes in the static gradient of entropy (or temperature if incompressible fluid is considered) at certain heights. In the solar convection zone, some prerequisites for such an effect are present, since the instability of the partial-ionisation layers of hydrogen and helium is enhanced. Some indirect indications of the possibility of scale splitting in such situations were found previously in the framework of a linear problem (Getling 1975, $1980)$; these expectations were partially substantiated by nonlinear numerical simulations (Getling \& Tikhomolov 2007).

Here, we numerically simulate two-dimensional convective motions in a horizontal layer of an incompressible fluid heated from below to study possibilities of the discretisation of the spectrum in the case where the static temperature gradient changes abruptly at some height. We specify a model temperature dependence of thermal diffusivity of the form $\chi(T)=1+a T+b T^{n}$ and appropriately choose the parameters $a, b$ and $n$ to obtain a static profile such that most part of the temperature difference, $\Delta T$, between the bottom and top surfaces of the layer of thickness $h$, which we denote as $(1-\delta T) \Delta T$, falls at a relatively thin sublayer of thickness $\delta T$, while, over the remaining part of the layer, whose thickness is $h-\delta h$, the temperature varies by a small quantity $\delta T \Delta T$ (Fig. 1 ). 
The horizontal size of the flow domain is equal to $5 \pi=15.7 \mathrm{~h}$ in our calculations. At all boundaries of the domain, we introduce no-slip condition, assume that the temperature is fixed at the horizontal boundaries and that the heat flux vanishes at the sidewalls.

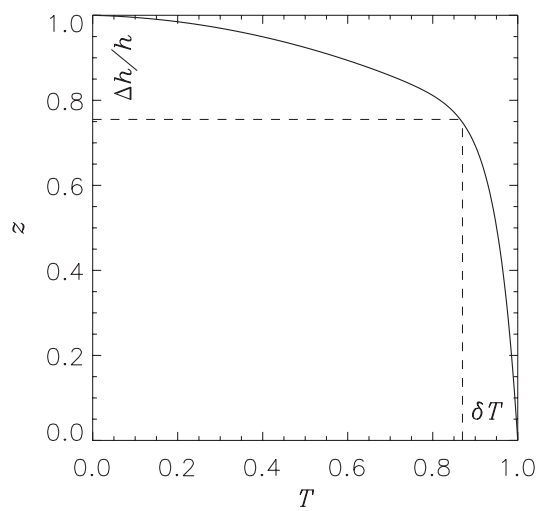

Figure 1. Static temperature profile.

The physical parameters of the problem are the Rayleigh and Prandtl numbers,

$$
\mathrm{Ra}=\frac{\beta g \Delta T h^{3}}{\nu \chi}, \quad \operatorname{Pr}=\frac{\nu}{\chi},
$$

where $\beta$ is the volumetric thermal-expansion coefficient of the fluid, $\nu$ and $\chi$ are its kinematic viscosity and thermal diffusivity (for the latter, the value at the upper layer boundary is used) and $g$ is the gravitational acceleration.

In the Boussinesq approximation, we solve the Navier-Stokes equations, written in terms of the stream function and vorticity, using a conservative finite-difference scheme developed by Mazhorova \& Popov (1980), of the second order in spatial coordinates and first order in time, for a nonuniform grid of $1024 \times 51$ points, which is finer near the top and bottom boundaries.

In the regimes studied, the critical Rayleigh number was typically $\mathrm{Ra}_{\mathrm{c}}=60000$; the computations were carried out for $\operatorname{Pr}=0.2,1$ and 100 .

The computed velocity fields were processed using four different techniques. We list them here together with the processing results. At $b=600$ in the ranges $a=10-20$ and $n=10-80$, these results in their qualitative features proved to be little sensitive to the choice of the parameters.

(1) The Fourier transform of the stream function in the horizontal coordinate at given heights indicates that, generally, there are spectral peaks that do not correspond to overtones of the fundamental mode and, therefore, reflect the presence of flows whose scales are not directly related to the fundamental scale.

(2) Running-average filtering was applied to the stream function, i.e., an average over a running window of one width or another was computed at a given height. Thus, a largescale component was selected in the flow, which was then subtracted from the original velocity field. At $\mathrm{Ra}=10 \mathrm{Ra}_{\mathrm{c}}, \operatorname{Pr}=100$, small-scale structures were identified in the bottom part of the layer; in the top part, they proved to be weaker and were lost in the process of averaging.

(3) The most interesting results were obtained by applying an ideal low-pass filter: the small-scale component was found by subtracting the principal spectral peak (which

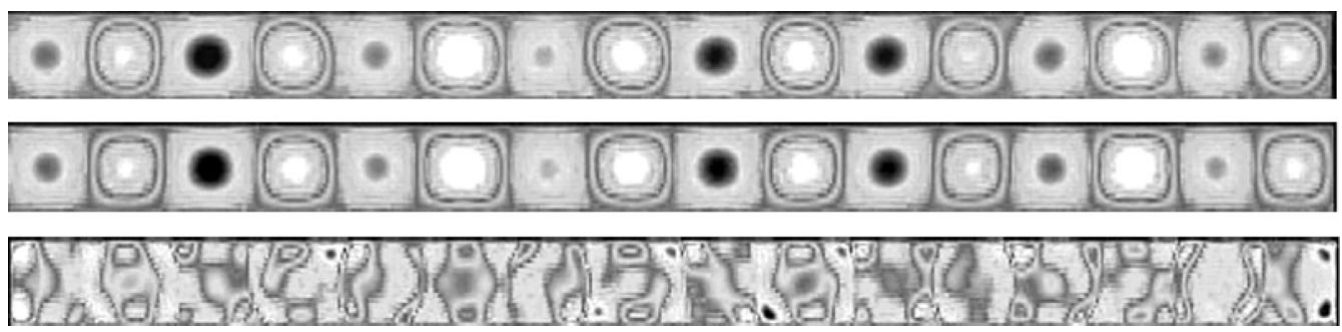

Figure 2. Velocity field found for $\mathrm{Ra} \approx 10 \mathrm{Ra}_{\mathrm{c}}, \operatorname{Pr}=1$ (top) and its large-scale (middle) and small-scale (bottom) components found by applying the ideal low-pass filter. Streamlines (contours of the stream function) are shown. 
corresponds to the fundamental mode) from the full spectrum of the flow. Figure 2 illustrates the case $\operatorname{Pr}=1$; the pattern is similar at $\operatorname{Pr}=0.2$, whereas larger cells near the bottom surface are observed ar $\operatorname{Pr}=100$.

(4) Signs of the presence of the small-scale component of the velocity field can visually be noted if we fill with black the regions in the vertical cross section of the layer where the vertical velocity component has a given sign (this is a preparatory procedure for applying the computational-homology techniques).

The results of our study can be formulated as follows:

- An abrupt change in the stratification parameters at a certain height near the layer boundary can give rise to small-scale flows and, on the whole, to the convection-scale splitting.

- Generally, small-scale cells are localised in both the upper and lower boundary layers; possibly, this means that the small-scale cells are carried by the large-scale flow.

- The thickness of the sublayer of small-scale convection cells is not directly related the thickness of the string-instability sublayer.

\section{Acknowledgement}

This work was supported by the Russian Foundation for Basic Research, project 12-02-00792-a.

\section{References}

Getling, A. V. 1975, Izv. Akad. Nauk SSSR, Mekhanika Zhidkosti i Gaza, no. 5, p. 45 (Fluid Dynamics, 10, 745, 1976)

Getling, A.V. 1980, Izv. Akad. Nauk SSSR, Fizika Atmosfery i Okeana, 16, 529 (Academy of Sciences, USSR, Izvestiya, Atmospheric and Oceanic Physics, 16, 363, 1980)

Getling, A. V. \& Tikhomolov, E. M. 2007 In Fizicheskaya pripoda solnechnoi aktivnosti i prognozirovanie ee geofizicheskikh proyavlenii (The Physical Nature of Solar Activity and Forecasting its Geophysical Manifestations), Proc. XI Pulkovo Int. Conf. on Solar Phys., 2-7 July 2007, Saint Petersburg, p. 109

Mazhorova, O. S. \& Popov, Yu. P. Zh. Vychislitel'noi Matematiki i Matematicheskoi Fiz., 20, 1005 